\title{
Analytical investigation of the two-dimensional cosmic ray Fokker-Planck equation
}

\author{
A. Shalchi \\ Institut für Theoretische Physik, Lehrstuhl IV: Weltraum und Astrophysik, Ruhr-Universität Bochum, 44780 Bochum, Germany \\ e-mail: andreasm4@yahoo.com \\ Received 20 June 2005 / Accepted 25 October 2005

\section{ABSTRACT} \\ The basic equation to describe transport of cosmic rays in a random magnetic field parallel to a magnetic background field is the two- \\ dimensional Fokker-Planck equation. In this paper an investigation of this equation is presented to improve understanding of particle transport, \\ to depict the process of parallel diffusion and to improve recently derived nonlinear transport theories.
}

Key words. cosmic rays - turbulence - diffusion

\section{Introduction}

The description of parallel and perpendicular diffusion of charged particles is central to cosmic ray astrophysics. Transport of such particles is described by diffusion coefficients that can be calculated by applying a transport theory. The knowledge of these coefficients is essential for describing solar energetic particles, the modulation of Galactic cosmic rays, and for the understanding of the measured abundance ratio of secondary to primary cosmic ray nuclei (for a review of applications see e.g. Schlickeiser 2002). The first theoretical description of parallel and perpendicular transport was achieved by the quasilinear theory (QLT, Jokipii 1966), which can be seen as a first-order perturbation theory in the small parameter $\delta B / B_{0}\left(\delta B\right.$ is the random magnetic field and $B_{0}$ is the mean field).

During the past few years it became successively clearer that a nonlinear description of cosmic ray transport has to be favored over the traditional quasilinear approach (see e.g. Shalchi et al. 2004; Shalchi 2005a). The key input into nonlinear transport theories is the so-called characteristic function

$\Gamma(\boldsymbol{k}, t)=\left\langle\mathrm{e}^{\mathrm{i} \boldsymbol{k} \cdot \boldsymbol{x}}\right\rangle=\int \mathrm{d}^{3} x f(\boldsymbol{x}, t) \mathrm{e}^{\mathrm{i} \boldsymbol{k} \cdot \boldsymbol{x}}$

with the particle distribution function $f$. Thus, it is essential to know the particle distribution function that can be obtained by solving the Fokker-Planck equation. Therefore a detailed discussion of the (two-dimensional) Fokker-Planck equation is presented in the current paper. Such an investigation can demonstrate in detail how parallel transport can be understood by a pitch-angle isotropization process. Furthermore, the results of the current paper could be important for improving nonlinear theories.
In Sect. 2, I consider the Fokker-Planck equation and the parallel spatial diffusion coefficient without specifying the pitch-angle Fokker-Planck coefficient that describes the interaction between the charged cosmic rays and the turbulent electromagnetic fields. A more detailed investigation of the Fokker-Planck equation is given in Sect. 3, where the isotropic form $D_{\mu \mu}=D \cdot\left(1-\mu^{2}\right)$ of the Fokker-Planck coefficient is assumed. While this assumption disagrees with results of quasilinear theory, I argue in Sect. 5 that this form seems to be reasonable within the recently derived second-order quasilinear theory (SOQLT, Shalchi 2005a). To derive concrete timedependent formulas for the parallel spatial diffusion coefficient and other parameters, such as the penetration depth, the case of sharp initial conditions is considered in Sect. 4. Despite the form of the pitch-angle Fokker-Planck coefficient assumed in Sects. 3 and 4, the Fokker-Planck equation itself must be justified. Both are done in Sect. 5 which is followed by Sect. 6 where, for completeness, the diffusion-transport equation is discussed.

\section{General investigation of the Fokker-Planck equation}

Starting from the relativistic Vlasov equation, it is straightforward to derive the six-dimensional Fokker-Planck equation (see e.g. Schlickeiser 2002)

$$
\begin{aligned}
& \frac{\partial f}{\partial t}+v \mu \frac{\partial f}{\partial z}-\Omega \frac{\partial f}{\partial \Phi}=S+\frac{1}{p^{2}} \frac{\partial}{\partial x_{\sigma}}\left(p^{2} D_{x_{\sigma} x_{v}} \frac{\partial f}{\partial x_{v}}\right), \\
& x_{\sigma}=x, y, z, \mu, \Phi, v .
\end{aligned}
$$

Here we used the pitch-angle cosine $\mu$, the gyrophase $\Phi$, the gyrofrequency $\Omega$, and the momentum of the particle $p$. 
The interaction between the charged cosmic rays and the fields is described by the Fokker-Planck coefficients $D_{x_{\sigma} x_{v}}$. To obtain the well-known two-dimensional Fokker-Planck equation, we neglect the source function $S$ and we average Eq. (2) over $x, y$ and the gyrophase $\Phi$. We also neglect momentum diffusion by assuming that $D_{p p}$ is very small to find finally:

$$
\frac{\partial f}{\partial t}+v \mu \frac{\partial f}{\partial z}=\frac{\partial}{\partial \mu}\left(D_{\mu \mu} \frac{\partial f}{\partial \mu}\right)
$$

The solution of this equation, $f=f(\mu, z, t)$, is the particle distribution function in the two-dimensional phase-space. In this equation the time-independent pitch-angle Fokker-Planck coefficient $D_{\mu \mu}$ was used. It was demonstrated in Shalchi (2005a) that there are small time-scales where pitch-angle transport has not reached diffusive behavior, the equation above cannot be applied for such small time-scales. This problem is discussed in Sect. 5. To proceed, we define the following functions:

$$
\begin{aligned}
F(\mu, t) & =\int_{-\infty}^{+\infty} \mathrm{d} z f(\mu, z, t) \\
\langle z\rangle(\mu, t) & =\int_{-\infty}^{+\infty} \mathrm{d} z z f(\mu, z, t) \\
\left\langle z^{2}\right\rangle(\mu, t) & =\int_{-\infty}^{+\infty} \mathrm{d} z z^{2} f(\mu, z, t) .
\end{aligned}
$$

By combining these definitions with Eq. (3), we obtain the following set of equations:

$$
\begin{array}{r}
\frac{\partial F}{\partial t}=\frac{\partial}{\partial \mu}\left(D_{\mu \mu} \frac{\partial F}{\partial \mu}\right) \\
\frac{\partial\langle z\rangle}{\partial t}-v \mu F=\frac{\partial}{\partial \mu}\left(D_{\mu \mu} \frac{\partial\langle z\rangle}{\partial \mu}\right) \\
\frac{\partial\left\langle z^{2}\right\rangle}{\partial t}-2 v \mu\langle z\rangle=\frac{\partial}{\partial \mu}\left(D_{\mu \mu} \frac{\partial\left\langle z^{2}\right\rangle}{\partial \mu}\right) .
\end{array}
$$

In turn we investigate these equations without specifying the form of $D_{\mu \mu}$.

\subsection{Relaxation time and pitch-angle isotropization}

Equation (5), in combination with the ansatz $F(\mu, t)=\mathrm{e}^{-\omega t}$. $P(\mu)$, can be rewritten as

$-\omega P_{l}(\mu)=\frac{\partial}{\partial \mu}\left(D_{\mu \mu} \frac{\partial P_{l}}{\partial \mu}\right)$.

The right side of this equation has the form of a SturmLivouville problem; therefore, the pitch-angle dependent functions $P_{l}(\mu)$ exist and form an orthogonal set. Thus the general (normalized) solution can be written as

$F(\mu, t)=1+\sum_{l=1}^{\infty} \alpha_{l} P_{l}(\mu) \mathrm{e}^{-\omega_{l} t}$.

The parameters $\alpha_{l}$ are determined by the initial conditions. Therefore the function $F$ becomes nearly isotropic after the time $\tau=\omega^{-1}$, which is called the pitch-angle relaxation timescale. This behavior is independent of the initial distribution function and of the form of the pitch-angle Fokker-Planck coefficient $D_{\mu \mu}$ that controls the form of the orthogonal functions $P_{l}$.

\subsection{Mean square deviation in the real-space}

The first step is that we consider the pitch-angle average of Eq. (7). Therefore we introduce the abbreviation

$$
\begin{aligned}
\langle A\rangle_{\mu}(t) & =\frac{1}{2} \int_{-1}^{+1} \mathrm{~d} \mu\langle A\rangle(\mu, t) \\
& =\frac{1}{2} \int_{-1}^{+1} \mathrm{~d} \mu \int_{-\infty}^{+\infty} \mathrm{d} z A(\mu, z, t) f(\mu, z, t) .
\end{aligned}
$$

Then in combination with Eq. (7) we obtain:

$\frac{\partial\left\langle z^{2}\right\rangle_{\mu}}{\partial t}-v \int_{-1}^{+1} \mathrm{~d} \mu \mu\langle z\rangle=\frac{1}{2} \int_{-1}^{+1} \mathrm{~d} \mu \frac{\partial}{\partial \mu}\left(D_{\mu \mu} \frac{\partial\left\langle z^{2}\right\rangle}{\partial \mu}\right)$.

The parameter $\left\langle z^{2}\right\rangle_{\mu}$ describes parallel spatial transport in the real-space. From the equations of motion, it follows directly (see e.g. Schlickeiser 2002, Eq. (12.1.9b)) that

$D_{\mu \mu}(\mu= \pm 1)=0$.

Thus the right side of Eq. (11) is equal to zero, so we obtain

$\frac{1}{2} \frac{\partial\left\langle z^{2}\right\rangle_{\mu}}{\partial t}=\frac{v}{2} \int_{-1}^{+1} \mathrm{~d} \mu \mu\langle z\rangle(\mu, t)$.

As shown later during the paper, the left hand side of this equation is exactly the parallel spatial diffusion coefficient in the real-space. To proceed we must calculate the parameter $\langle z\rangle(\mu, t)$, which is done in turn.

\subsection{The mean position in the phase-space}

To determine the parameter $\langle z\rangle(\mu, t)$, we apply the operator

$\int_{-1}^{v} \mathrm{~d} \mu$

onto Eq. (6):

$\frac{\partial}{\partial t} \int_{-1}^{v} \mathrm{~d} \mu\langle z\rangle(\mu, t)-v \int_{-1}^{v} \mathrm{~d} \mu \mu F(\mu, t)=D_{v v} \frac{\partial\langle z\rangle(v, t)}{\partial v}$,

where again we used Eq. (12). To proceed, we assume that the distribution function becomes isotropic for large time-scales $F(\mu, t) \approx 1$ (see Sect. 2.1). Thus we obtain

$\frac{\partial}{\partial t} \int_{-1}^{v} \mathrm{~d} \mu\langle z\rangle(\mu, t)+\frac{v}{2}\left(1-v^{2}\right)=D_{v v} \frac{\partial\langle z\rangle(v, t)}{\partial v}$.

Because of the pitch-angle isotropization process, we can also assume that $\langle z\rangle(\mu, t)$ becomes time-independent for large enough times, so we find

$\frac{v}{2} \frac{\left(1-\mu^{2}\right)}{D_{\mu \mu}}+\frac{\partial}{\partial \mu}\langle z\rangle(\mu)=0$,

which has the solution

$\langle z\rangle(\mu)=\langle z\rangle(\mu=-1)+\frac{v}{2} \int_{-1}^{\mu} \mathrm{d} v \frac{\left(1-v^{2}\right)}{D_{v v}}$.

This result can be combined with Eq. (13) to determine the parallel spatial diffusion coefficient, as below. 


\subsection{The parallel spatial diffusion coefficient}

With Eqs. (13) and (18) we have

$$
\begin{aligned}
\frac{1}{2} \frac{\partial\left\langle z^{2}\right\rangle_{\mu}}{\partial t}= & \frac{v}{2} \int_{-1}^{+1} \mathrm{~d} \mu \mu\langle z\rangle(\mu=-1) \\
& +\frac{v^{2}}{4} \int_{-1}^{+1} \mathrm{~d} \mu \mu \int_{-1}^{\mu} \mathrm{d} v \frac{\left(1-v^{2}\right)}{D_{v v}} .
\end{aligned}
$$

The first term on the right hand side is equal to zero and can be dropped. The second term can be written as

$$
\begin{aligned}
\frac{1}{2} \frac{\partial\left\langle z^{2}\right\rangle_{\mu}}{\partial t} & =\frac{v^{2}}{8} \int_{-1}^{+1} \mathrm{~d} \mu \frac{\partial \mu^{2}}{\partial \mu} \int_{-1}^{\mu} \mathrm{d} v \frac{\left(1-v^{2}\right)}{D_{v v}} \\
& =\frac{v^{2}}{8}\left[\mu^{2} \int_{-1}^{\mu} \mathrm{d} v \frac{1-v^{2}}{D_{v v}}\right]_{-1}^{+1}-\frac{v^{2}}{8} \int_{-1}^{+1} \mathrm{~d} \mu \mu^{2} \frac{\left(1-\mu^{2}\right)}{D_{\mu \mu}} \\
& =\frac{v^{2}}{8} \int_{-1}^{+1} \mathrm{~d} \mu \frac{1-\mu^{2}}{D_{v v}}-\frac{v^{2}}{8} \int_{-1}^{+1} \mathrm{~d} \mu \mu^{2} \frac{\left(1-\mu^{2}\right)}{D_{\mu \mu}} \\
& =\frac{v^{2}}{8} \int_{-1}^{+1} \mathrm{~d} \mu \frac{\left(1-\mu^{2}\right)^{2}}{D_{\mu \mu}} .
\end{aligned}
$$

The assumption

$$
\left\langle z^{2}\right\rangle_{\mu}=2 t \kappa_{\|}
$$

with the spatial parallel diffusion coefficient

$\kappa_{\|}=\frac{v^{2}}{8} \int_{-1}^{+1} \mathrm{~d} \mu \frac{\left(1-\mu^{2}\right)^{2}}{D_{\mu \mu}}$

seems to be reasonable. This result was already derived in Earl (1974) by using a different approach based on the same physical assumptions, which are:

1) the Fokker-Planck equation provides the correct description of particle transport in the phase-space;

2) the particle distribution function $f(\mu, t)$ becomes isotropic in the pitch-angle for large time-scales, and thus $F(\mu, t \rightarrow$ $\infty) \rightarrow 1$ and $\langle z\rangle(\mu, t \rightarrow \infty) \rightarrow\langle z\rangle(\mu)$.

\section{Investigation of the Fokker-Planck equation for an isotropic form of $D_{\mu \mu}$}

In Sect. 2 the Fokker-Planck equation was discussed without specifying the form of the pitch-angle Fokker-Planck coefficient $D_{\mu \mu}$. To obtain a more understandable description of cosmic ray transport, and because of the discussions in Sect. 5, we assume

$D_{\mu \mu}=D \cdot\left(1-\mu^{2}\right)$

for the pitch-angle Fokker-Planck coefficient. In this case Eq. (5) becomes

$\frac{\partial F}{\partial t}=\frac{\partial}{\partial \mu}\left(D_{\mu \mu} \frac{\partial F}{\partial \mu}\right)=D \frac{\partial}{\partial \mu}\left(\left(1-\mu^{2}\right) \frac{\partial F}{\partial \mu}\right)$.
By applying the ansatz $F(\mu, t)=\rho(t) \cdot P(\mu)$, we find

$\frac{\partial \rho}{\partial t}=-\omega \rho(t)$

$\left(1-\mu^{2}\right) \frac{\partial^{2} P}{\partial \mu^{2}}-2 \mu \frac{\partial P}{\partial \mu}+\frac{\omega}{D} F=0$.

For the function $\rho$ we immediately find an exponential law

$\rho(t)=\mathrm{e}^{-\omega t}$.

In the second equation we must have

$\frac{\omega}{D}=l(l+1), \quad l=0,1,2,3, \ldots$

to prevent a singularity, and we find

$\left(1-\mu^{2}\right) \frac{\partial^{2} P_{l}}{\partial \mu^{2}}-2 \mu \frac{\partial P_{l}}{\partial \mu}+l(l+1) P_{l}=0$.

In this case we have a differential equation with a well-known solution: the functions $P_{l}(\mu)$ can be identified with LegendrePolynoms. The general solution of Eq. (24) is therefore

$F(\mu, t)=\sum_{l=0}^{\infty} \alpha_{l} P_{l}(\mu) \mathrm{e}^{-l(l+1) D t}$.

This form agrees with considerations of Sect. 2.1, which are based on the Sturm-Liouville theory. Now we assume that the function $F(\mu, t)$ is normalized:

$$
\begin{aligned}
1 & =\frac{1}{2} \int_{-1}^{+1} \mathrm{~d} \mu F(\mu, t) \\
& =\frac{1}{2} \sum_{l=0}^{\infty} \alpha_{l} \mathrm{e}^{-l(l+1) D t} \int_{-1}^{+1} \mathrm{~d} \mu P_{l}(\mu) \\
& =\alpha_{0}
\end{aligned}
$$

because we have

$\int_{-1}^{+1} \mathrm{~d} \mu P_{l}(\mu) P_{m}(\mu)=\frac{2}{2 m+1} \delta_{l m}$

and $P_{0}(\mu)=1$. Therefore we find

$F(\mu, t)=1+\sum_{l=1}^{\infty} \alpha_{l} P_{l}(\mu) \mathrm{e}^{-l(l+1) D t}$.

The other parameters $\alpha_{l}$ can be determined by the initial conditions:

$\alpha_{l}=\frac{2 l+1}{2} \int_{-1}^{+1} \mathrm{~d} \mu F(\mu, 0) P_{l}(\mu)$.

\subsection{The relaxation time-scale}

If we consider large time-scales $2 D t \gg 1$, we have approximately

$F(\mu, t) \approx 1+\alpha_{1} P_{1}(\mu) \mathrm{e}^{-2 D t}=1+\alpha_{1} \mu \mathrm{e}^{-t / \tau}$

with the relaxation time

$\tau=\frac{1}{2 D}=\frac{1-\mu^{2}}{2 D_{\mu \mu}}$. 
Analogously one could define the scattering frequency as $\omega=\tau^{-1}$. Independent of the initial conditions $\left(\alpha_{l}\right)$, we find for time-scales much larger than the relaxation time:

$F(\mu, t \gg \tau) \approx 1$.

The particle distribution function becomes nearly isotropic after the time $\tau$.

\subsection{Parameters in the real-space}

Here we calculate different parameters in the real-space by considering the total average defined in Eq. (10). First we consider $A=\mu$ to determine the time-behavior of the mean pitch-angle cosine in the real-space:

$$
\begin{aligned}
\langle\mu\rangle_{\mu} & =\frac{1}{2} \int_{-1}^{+1} \mathrm{~d} \mu \mu F(\mu, t) \\
& =\frac{1}{2} \int_{-1}^{+1} \mathrm{~d} \mu \mu+\frac{1}{2} \sum_{l=1}^{\infty} \alpha_{l} \mathrm{e}^{-l(l+1) D t} \int_{-1}^{+1} \mathrm{~d} \mu \mu P_{l}(\mu) \\
& =\frac{1}{2} \sum_{l=1}^{\infty} \alpha_{l} \mathrm{e}^{-l(l+1) D t} \int_{-1}^{+1} \mathrm{~d} \mu P_{1}(\mu) P_{l}(\mu)
\end{aligned}
$$

because of $\mu=P_{1}(\mu)$. By using Eq. (32) we find

$\langle\mu\rangle_{\mu}=\frac{\alpha_{1}}{3} \mathrm{e}^{-t / \tau}$.

After the relaxation time, the average pitch-angle in the realspace tends to zero, independent of the initial conditions $\left(\alpha_{1}\right)$. Also the parameter $\mu^{2}$ can be averaged in the same manner. By using

$\mu^{2}=\frac{2}{3} P_{2}(\mu)+\frac{1}{3} P_{0}(\mu)$,

we obtain

$$
\begin{aligned}
\left\langle\mu^{2}\right\rangle_{\mu} & =\frac{1}{2} \int_{-1}^{+1} \mathrm{~d} \mu \mu^{2} F(\mu, t) \\
& =\frac{1}{2} \int_{-1}^{+1} \mathrm{~d} \mu \mu^{2}+\frac{1}{2} \sum_{l=1}^{\infty} \alpha_{l} \mathrm{e}^{-l(l+1) D t} \int_{-1}^{+1} \mathrm{~d} \mu \mu^{2} P_{l}(\mu) \\
& =\frac{1}{3}+\frac{1}{3} \sum_{l=1}^{\infty} \alpha_{l} \mathrm{e}^{-l(l+1) D t} \int_{-1}^{+1} \mathrm{~d} \mu P_{2}(\mu) P_{l}(\mu) \\
& =\frac{1}{3}+\frac{2}{15} \alpha_{2} \mathrm{e}^{-6 D t} .
\end{aligned}
$$

For times much larger than $\tau$, we find $\left\langle\mu^{2}\right\rangle_{\mu} \approx 1 / 3$, which is the expected isotropic result. In the last step we determine the parameter $\langle z\rangle_{\mu}$ as the average position in the real-space. To do this we consider the pitch-angle average of Eq. (6)

$$
\begin{aligned}
\frac{\partial\langle z\rangle_{\mu}}{\partial t} & =\frac{v}{2} \int_{-1}^{+1} \mathrm{~d} \mu(\mu F)+\frac{1}{2}\left[D_{\mu \mu} \frac{\partial\langle z\rangle}{\partial \mu}\right]_{-1}^{+1} \\
& =\frac{v}{2} \int_{-1}^{+1} \mathrm{~d} \mu \mu \sum_{l=1}^{\infty} \alpha_{l} P_{l}(\mu) \mathrm{e}^{-l(l+1) D t} \\
& =\frac{v}{2} \sum_{l=1}^{\infty} \alpha_{l} \mathrm{e}^{-l(l+1) D t} \int_{-1}^{+1} \mathrm{~d} \mu P_{1}(\mu) P_{l}(\mu) \\
& =\frac{v}{3} \alpha_{1} \mathrm{e}^{-t / \tau}
\end{aligned}
$$

and therefore

$\langle z\rangle_{\mu}(t)=$ const. $-\frac{v \tau \alpha_{1}}{3} \mathrm{e}^{-t / \tau}$.

By assuming $\langle z\rangle_{\mu}(0)=0$, we obtain

$\langle z\rangle_{\mu}(t)=z_{\mathrm{PD}} \cdot\left(1-\mathrm{e}^{-t / \tau}\right)$

with the penetration depth

$z_{\mathrm{PD}}=\frac{v \tau \alpha_{1}}{3}=\frac{v}{6 D} \alpha_{1}$,

which is dependent of the initial conditions $\left(\alpha_{1}\right)$. These results are discussed for sharp initial conditions in Sect. 4.

\subsection{The time-dependent parallel diffusion coefficient}

Here we calculate the time-dependent parallel diffusion coefficient:

$\kappa_{\|}(t):=\frac{1}{2 t}\left\langle z^{2}\right\rangle_{\mu}(t)$

From Eq. (13) we know the exact relation

$\frac{\partial\left\langle z^{2}\right\rangle_{\mu}(t)}{\partial t}=v \int_{-1}^{+1} \mathrm{~d} \mu \mu\langle z\rangle(\mu, t)=2 v\langle\mu z\rangle_{\mu}(t)$,

and from Eq. (6) we obtain

$$
\begin{aligned}
\frac{\partial\langle\mu z\rangle_{\mu}(t)}{\partial t}= & \frac{v}{2} \int_{-1}^{+1} \mathrm{~d} \mu \mu^{2} F(\mu, t) \\
& +\frac{1}{2} \int_{-1}^{+1} \mathrm{~d} \mu \mu \frac{\partial}{\partial \mu}\left(D_{\mu \mu} \frac{\partial}{\partial \mu}\langle z\rangle\right) \\
= & \frac{v}{2} \int_{-1}^{+1} \mathrm{~d} \mu \mu^{2} F(\mu, t)-\frac{1}{2} \int_{-1}^{+1} \mathrm{~d} \mu D_{\mu \mu} \frac{\partial}{\partial \mu}\langle z\rangle \\
= & \frac{v}{2} \int_{-1}^{+1} \mathrm{~d} \mu \mu^{2} F(\mu, t)-2 D\langle\mu z\rangle_{\mu}(t) .
\end{aligned}
$$

With Eqs. (30) and (40) we find

$$
\begin{aligned}
\frac{v}{2} \int_{-1}^{+1} \mathrm{~d} \mu \mu^{2} F(\mu, t) & =\frac{v}{3}+\frac{v}{3} \sum_{l=1}^{\infty} \alpha_{l} \mathrm{e}^{-l(l+1) D t} \int_{-1}^{+1} \mathrm{~d} \mu P_{l}(\mu) P_{2}(\mu) \\
& =\frac{v}{3}+\frac{2 v}{15} \alpha_{2} \mathrm{e}^{-6 D t}
\end{aligned}
$$

and therefore

$\frac{\partial\langle\mu z\rangle_{\mu}(t)}{\partial t}+2 D\langle\mu z\rangle_{\mu}(t)=\frac{v}{3}+\frac{2 v}{15} \alpha_{2} \mathrm{e}^{-6 D t}$.

By using

$$
\begin{aligned}
\kappa_{\|, \infty} & =\frac{v^{2}}{8} \int_{-1}^{+1} \mathrm{~d} \mu \frac{\left(1-\mu^{2}\right)^{2}}{D_{\mu \mu}} \\
& =\frac{v^{2}}{8 D} \int_{-1}^{+1} \mathrm{~d} \mu\left(1-\mu^{2}\right)=\frac{v^{2}}{6 D}
\end{aligned}
$$

and

$\lambda_{\|, \infty}=\frac{3}{v} \kappa_{\|, \infty}=\frac{v}{2 D}$ 
we have

$\frac{\partial\langle\mu z\rangle_{\mu}(t)}{\partial t}+\frac{v}{\lambda_{\|, \infty}}\langle\mu z\rangle_{\mu}(t)=\frac{v}{3}+\frac{2 v}{15} \alpha_{2} \mathrm{e}^{-3 v t / \lambda_{\|, \infty}}$.

This differential equation has the homogenous solution

$\langle\mu z\rangle_{\mu}(t)=\beta_{1} \mathrm{e}^{-v t / \lambda_{\|, \infty}}$

with the constant $\beta_{1}$ and the particular solution

$\langle\mu z\rangle_{\mu}(t)=\frac{\lambda_{\|, \infty}}{3}-\frac{\lambda_{\|, \infty}}{15} \alpha_{2} \mathrm{e}^{-3 v t / \lambda_{\|, \infty}}$.

The general solution is simply a superposition of Eqs. (54) and (55)

$\langle\mu z\rangle_{\mu}(t)=\beta_{1} \mathrm{e}^{-v t / \lambda_{\|, \infty}}+\frac{\lambda_{\|, \infty}}{3}-\frac{\lambda_{\|, \infty}}{15} \alpha_{2} \mathrm{e}^{-3 v t / \lambda_{\|, \infty}}$.

With this result Eq. (47) becomes

$$
\begin{aligned}
\frac{\partial}{\partial t}\left\langle z^{2}\right\rangle_{\mu}(t) & =2 \kappa_{\|, \infty}+2 v \beta_{1} \mathrm{e}^{-v^{2} t /\left(3 \kappa_{\|, \infty}\right)} \\
& -\frac{2 \kappa_{\|, \infty}}{5} \alpha_{2} \mathrm{e}^{-v^{2} t / \kappa_{\|, \infty}}
\end{aligned}
$$

and we therefore find

$$
\begin{aligned}
\kappa_{\|}(t)= & \frac{\beta_{2}}{2 t}+\kappa_{\|, \infty}-\frac{3 \beta_{1} \kappa_{\|, \infty}}{v t} \mathrm{e}^{-v^{2} t /\left(3 \kappa_{\|, \infty}\right)} \\
& +\frac{\alpha_{2} \kappa_{\|, \infty}^{2}}{5 v^{2} t} \mathrm{e}^{-v^{2} t / \kappa_{\|, \infty}} .
\end{aligned}
$$

This result justifies the definition of $\kappa_{\|, \infty}$, because in the limit $t \rightarrow \infty$ we have

$\lim _{t \rightarrow \infty} \kappa_{\|}(t)=\kappa_{\|, \infty}$

and we find diffusive behavior in agreement with the more general derivation of Eq. (22). The other contributions in Eq. (58) are only important for small time-scales $\left(v t \ll \lambda_{\|, \infty}\right)$. The parameters $\alpha_{2}, \beta_{1}, \beta_{2}$ can be determined by the initial conditions. A concrete visualization of the time behavior of all calculated parameters can only be performed if we specify the initial conditions which in done in Sect. 4.

\subsection{The diffusion-transport-equation}

So far, we have considered the $z$-average of the Fokker-Planck equation. In contrast to the previous discussions, we now consider the $\mu$-average. By defining the parameters

$M(z, t)=\frac{1}{2} \int_{-1}^{+1} \mathrm{~d} \mu f(\mu, z, t)$
$N(z, t)=\frac{1}{2} \int_{-1}^{+1} \mathrm{~d} \mu \mu f(\mu, z, t)$
$P(z, t)=\frac{1}{2} \int_{-1}^{+1} \mathrm{~d} \mu \mu^{2} f(\mu, z, t)$

we find the following equations:

$$
\begin{aligned}
& \frac{\partial M}{\partial t}+v \frac{\partial N}{\partial z}=0 \\
& \frac{\partial N}{\partial t}+v \frac{\partial P}{\partial z}=-\frac{1}{2} \int_{-1}^{+1} \mathrm{~d} \mu D_{\mu \mu} \frac{\partial f}{\partial \mu} .
\end{aligned}
$$

Since

$\frac{\partial D_{\mu \mu}}{\partial \mu}=-2 \mu D$

we find for Eq. (62)

$\frac{\partial N}{\partial t}+v \frac{\partial P}{\partial z}=-2 D N$

Calculating the $z$-derivative of this equation and applying Eq. (61), we obtain

$\frac{\partial^{2} N}{\partial t \partial z}+v \frac{\partial^{2} P}{\partial z^{2}}=-2 D \frac{\partial N}{\partial z}=\frac{2 D}{v} \frac{\partial M}{\partial t}$.

To proceed we consider the limit $t \rightarrow \infty$. Because of the previous discussions we have

$N(t \rightarrow \infty) \rightarrow 0$

and

$P(t \rightarrow \infty) \rightarrow \frac{M}{3}$

Thus we finally find

$\frac{\partial M}{\partial t}=\kappa_{z z} \frac{\partial^{2} M}{\partial z^{2}}$

with $\kappa_{z z}=\kappa_{\|}=v^{2} /(6 D)$. As expected we find a diffusion equation as transport equation. Solutions to this equations and its three-dimensional generalization are discussed in Sect. 6.

\section{Sharp initial conditions}

For a further simplification we assume sharp initial conditions:

$$
\begin{aligned}
z(0) & =0 \\
\mu(0) & =\mu_{0} \\
F(\mu, t=0) & =2 \delta\left(\mu-\mu_{0}\right)
\end{aligned}
$$

and we have

$$
\begin{aligned}
\alpha_{l} & =\frac{2 l+1}{2} \int_{-1}^{+1} \mathrm{~d} \mu F(\mu, 0) P_{l}(\mu) \\
& =(2 l+1) \int_{-1}^{+1} \mathrm{~d} \mu \delta\left(\mu-\mu_{0}\right) P_{l}(\mu) \\
& =(2 l+1) P_{l}\left(\mu_{0}\right)
\end{aligned}
$$

and therefore

$F(\mu, t)=1+\sum_{l=1}^{\infty}(2 l+1) P_{l}\left(\mu_{0}\right) P_{l}(\mu) \mathrm{e}^{-l(l+1) D t}$.

Figure 1 shows the function $F(\mu, t)$ for $\mu_{0}=1 / \sqrt{2}$ for different times. For very short time-scales $\left(t / \tau \sim 10^{-2}\right)$, we find a distribution function that is very similar to the initial state $\left(F=2 \delta\left(\mu-\mu_{0}\right)\right)$. For larger times $\left(t / \tau \sim 10^{-1}\right)$ the broadening of the distribution function becomes larger. For $t / \tau \sim 1$ we already find a nearly isotropic distribution function, and for timescales much larger than the relaxation time, we find $F \approx 1$. 


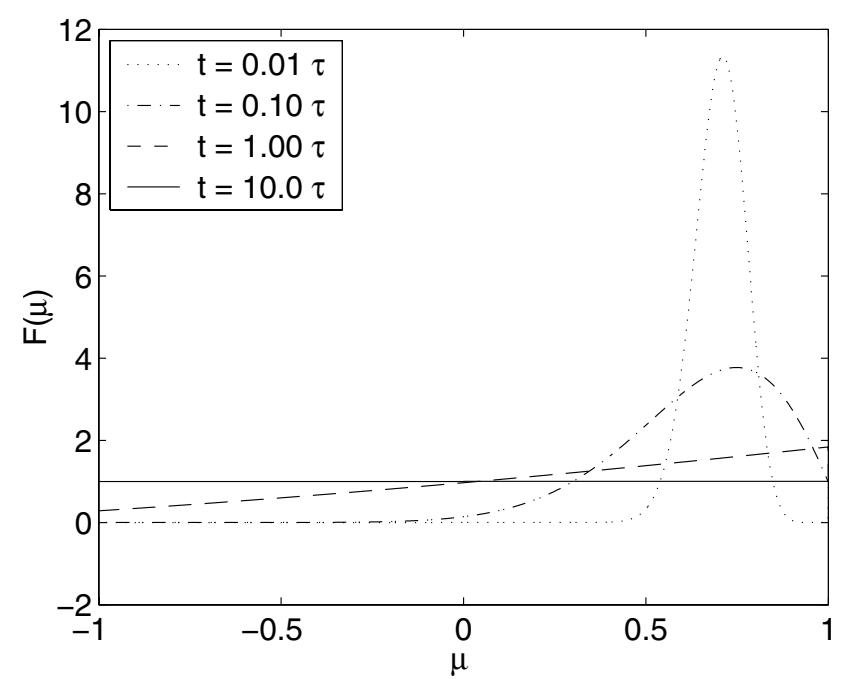

Fig. 1. Shown is the function $F(\mu)$ for different values of the time: $t / \tau=0.01,0.1,1,10$ to visualize the pitch-angle isotropization process. Here we assume that $\mu(t=0)=\mu_{0}=1 / \sqrt{2}$.

\subsection{Parameters in the real-space for sharp initial conditions}

Here we reconsider the parameters calculated in Sect. 3.2 for sharp initial conditions. With

$\alpha_{1}=3 P_{1}\left(\mu_{0}\right)=3 \mu_{0}$

we obtain with Eq. (39) the exact formula

$\langle\mu\rangle_{\mu}=\mu_{0} \mathrm{e}^{-t / \tau}$.

The penetration depth is

$z_{\mathrm{PD}}=\frac{v \mu_{0}}{2 D}=\mu_{0} \lambda_{\|, \infty}$,

and for $t \gg \tau$ we have $\lambda_{\|}=\lambda_{\|, \infty}$ and therefore

$\langle z\rangle_{\mu}(t)=z_{\mathrm{PD}}=\mu_{0} \lambda_{\|}$

We find the maximal penetration depth if $\mu_{0} \pm 1$, so we then have $z_{\mathrm{PD}}= \pm \lambda_{\|}$.

\subsection{The parallel diffusion coefficient for sharp initial conditions}

Here we consider Eq. (58) for the case of sharp initial conditions. First we assume that $\left\langle z^{2}\right\rangle_{\mu}(t=0)=0$ and $\langle\mu z\rangle_{\mu}(t=0)=0$ to have $\beta_{2}=0$ and

$\beta_{1}=\frac{\alpha_{2}-5}{15} \lambda_{\|, \infty}$

With

$\alpha_{2}=5 P_{2}\left(\mu_{0}\right)=\frac{15}{2} \mu_{0}^{2}-\frac{5}{2}$

we obtain

$\beta_{1}=\frac{1}{2} \lambda_{\|, \infty}\left(\mu_{0}^{2}-1\right)$

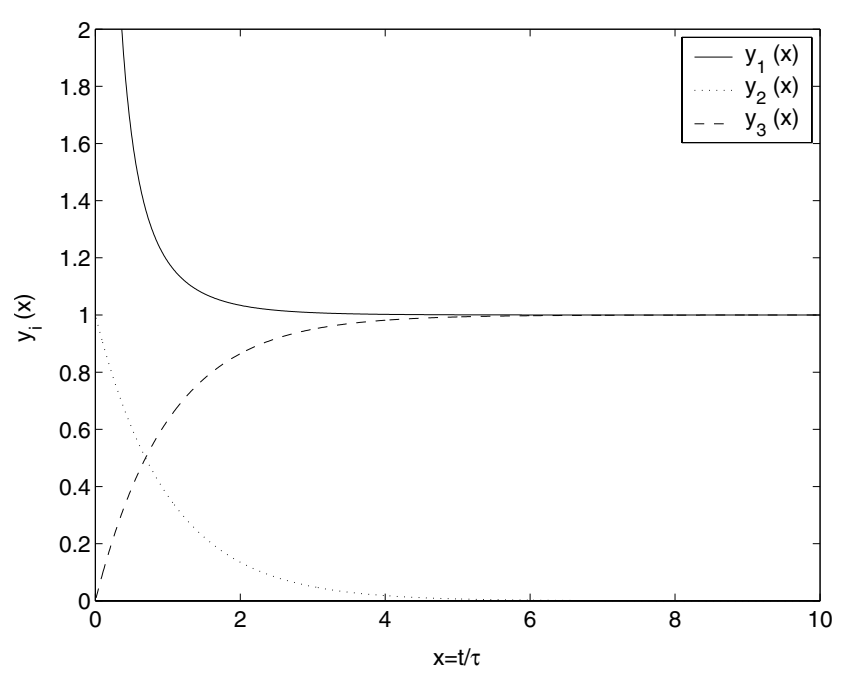

Fig. 2. The time-dependence of the parallel diffusion coefficient $y_{1}(x)$ (solid line), the mean pitch-angle cosine $y_{2}(x)$ (dotted line), and the mean position $y_{3}(x)$ (dashed line).

and we finally find for the time-dependent parallel diffusion coefficient

$$
\begin{aligned}
\kappa_{\|}(t)= & \kappa_{\|, \infty}+\frac{\lambda_{\|, \infty}^{2}}{2 t}\left(1-\mu_{0}^{2}\right) \mathrm{e}^{-v t / \lambda_{\|, \infty}} \\
& +\frac{\lambda_{\|, \infty}^{2}}{6 t}\left(\mu_{0}^{2}-\frac{1}{3}\right) \mathrm{e}^{-3 v t / \lambda_{\|, \infty}}
\end{aligned}
$$

In Fig. 2 the time-dependence of the parallel diffusion coefficient and other parameters are shown for:

$$
\begin{aligned}
x & =t / \tau \\
y_{1}(x) & =\frac{2}{v^{2} \tau} \kappa_{\|}(t) \\
y_{2}(x) & =\frac{\langle\mu\rangle_{\mu}}{\mu_{0}}=\mathrm{e}^{-x} \\
y_{3}(x) & =\frac{\langle z\rangle_{\mu}}{z_{\mathrm{PD}}}=1-\mathrm{e}^{-x} \\
\mu_{0} & =\frac{1}{\sqrt{2}} \\
\frac{2}{v^{2} \tau} \kappa_{\|, \infty} & =1 .
\end{aligned}
$$

After a certain time-scale (relaxation-time), parallel transport reaches diffusive behavior, the mean pitch-angle goes to zero $\left(y_{2} \rightarrow 0\right)$, and the mean parallel position reaches the penetration depth $\left(y_{3} \rightarrow 1\right)$.

\section{Validity of the presented results}

During the last sections two assumptions were made that were not justified: we considered the Fokker-Planck equation with a time-independent pitch-angle Fokker-Planck coefficient $D_{\mu \mu}$, and assumed the form $D_{\mu \mu}=D \cdot\left(1-\mu^{2}\right)$. In turn we now discuss both assumptions to examine the validity of our results. 


\subsection{Validity of the Fokker-Planck equation}

Within the quasilinear approach, it is straightforward to determine the time-dependent pitch-angle Fokker-Planck coefficient

$D_{\mu \mu}(\mu, t)=\frac{\left\langle(\Delta \mu)^{2}\right\rangle}{2 t}$.

The usual time-independent coefficent $D_{\mu \mu}(\mu, t)$ can be obtained by the limit

$D_{\mu \mu}(\mu)=\lim _{t \rightarrow \infty} D_{\mu \mu}(\mu, t)$.

To proceed we define the time-scale $t_{\mu}$ as:

$D_{\mu \mu}(\mu) \approx D_{\mu \mu}\left(\mu, t \gg t_{\mu}\right)$.

For smaller time-scales than $t_{\mu}$, the time-dependence of $D_{\mu \mu}$ cannot be neglected. If we consider the limit $t \gg \tau, t_{\mu}$, we should obtain diffusive behavior of $D_{\mu \mu}$ and $\kappa_{\|}$. For time-scales where $t_{\mu} \ll t \ll \tau$, we can use the results of the current paper (see Sects. 3 and 4). If there are time-scales with $\tau \ll t \ll t_{\mu}$ where we find a nearly isotropic pitch-angle distribution, but pitch-angle scattering has not reached diffusive behavior, the results of the current paper cannot be applied, and the timedependence shown in Shalchi (2005b; and Fig. 1) should be valid. Numerical test particle simulations provide a reasonable possibility for investigating such different time-scales.

\subsection{Validity of the Fokker-Planck coefficient}

The quasilinear pitch-angle Fokker-Planck coefficient for pure magnetostatic slab turbulence has the form

$D_{\mu \mu}=D \cdot\left(1-\mu^{2}\right) \cdot|\mu|^{s-1}$

with the inertial range spectral index $s$. For $90^{\circ}(\mu=0)$ we find a vanishing Fokker-Planck coefficient within the quasilinear theory. According to Shalchi (2005a), nonlinear effects are important if we consider pitch-angle diffusion close to $90^{\circ}$ in strong or medium strong turbulence $\left(\delta B \geq B_{0}\right)$. Within the recently proposed second order QLT (SOQLT, Shalchi 2005a), it is also possible to describe pitch-angle transport close to $90^{\circ}$. Figure 3 shows the quasilinear result, the SOQLT-result, and a $\left(1-\mu^{2}\right)$-fit. It is obvious that the fit agrees with the SOQLT result very well. Therefore the form of Eq. (23) should be appropriate if we assume that turbulence is not too weak . A formula for $D=D_{\mu \mu}(\mu=0)$ is given in Shalchi (2005a). Even in cases where the form of Eq. (23) is not accurate (e.g. very weak turbulence), the results of Sect. 3 should at least be qualitatively correct. It should also be noted that the formulas for $D_{\mu \mu}$ discussed here (e.g. Eq. (84)) are only valid for magnetostatic turbulence. More realistic turbulence models are available and are discussed e.g. in Schlickeiser (2002). Prominent examples are the plasmawave model or the dynamical turbulence model. In general such models can change the form of a Fokker-Planck coefficient; e.g., Chandran (2000) notes that for Alfvénic turbulence, $D_{\mu \mu}$ is anisotropic. But such conclusions are based on quasilinear theory. Within a nonlinear description one would expect a form of $D_{\mu \mu}$ that is at least nearly isotropic. A computation of $D_{\mu \mu}$ for more realistic models, such as Alfvénic turbulence, is the subject of future work.

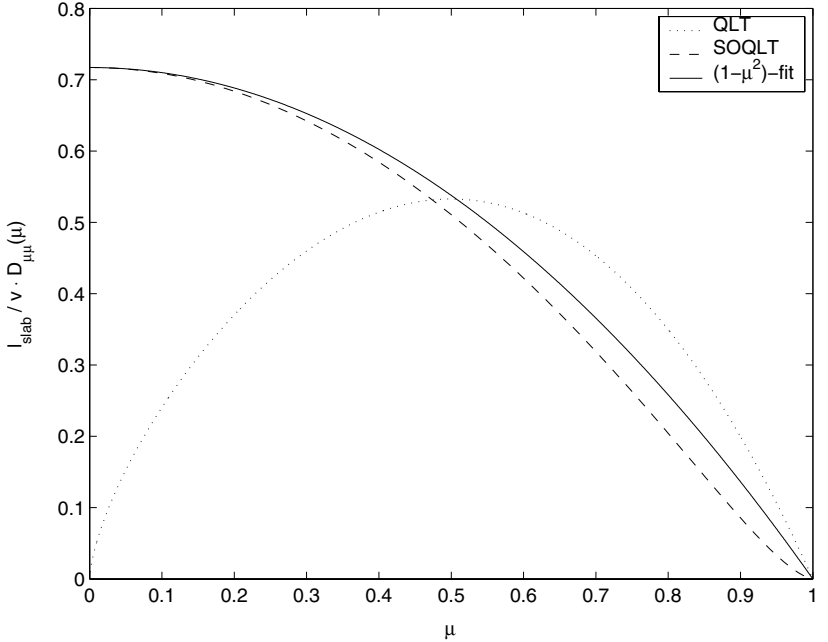

Fig. 3. The QLT result (dotted line), the SOQLT result (dashed line), and a $\left(1-\mu^{2}\right)$-fit (solid line) for the pitch-angle Fokker-Planck coefficient $D_{\mu \mu}$ as a function of the pitch-angle cosine $\mu$.

\section{Investigation of the diffusion-transport-equation}

In Eq. (68) the diffusion-transport-equation was derived for the isotropic form of $D_{\mu \mu}$. As shown e.g. in Schlickeiser (2002) the general diffusion-transport-equation has the form

$\frac{\partial M}{\partial t}=\kappa_{z z} \frac{\partial^{2} M}{\partial z^{2}}+\sum_{i, j=x, y} \kappa_{i j} \frac{\partial^{2} M}{\partial x_{i} \partial x_{j}}$

if we neglect momentum diffusion. In the case of axisymmetric turbulence we have $\kappa_{x y}=\kappa_{y x}=0$ and therefore

$\frac{\partial M}{\partial t}=\sum_{i=x, y, z} \kappa_{i i} \frac{\partial^{2} M}{\partial x_{i}^{2}}$.

In the $\boldsymbol{k}$-space this equation becomes

$\frac{\partial M\left(k_{j}\right)}{\partial t}=-\sum_{i=x, y, z} k_{i}^{2} \kappa_{i i} M\left(k_{j}\right)$

and we find the particular solution

$M\left(k_{j}\right)=\mathrm{e}^{-\sum_{i=x, y, z} k_{i}^{2} \kappa_{i i} t}$.

In general the solution is a superposition of the different particular solutions, but if we assume Gaussian statistics, the last equation is already the correct solution to the transport equation. In the real-space we have

$M(x, y, z)=\frac{1}{(2 \pi)^{3 / 2} \sqrt{8 t^{3} \kappa_{x x} K_{y y} K_{z z}}} \mathrm{e}^{-\frac{x^{2}}{4 t k_{x x}}-\frac{y^{2}}{4 \kappa_{y y y}}-\frac{z^{2}}{4 t k_{z z}}}$.

We define the average of the parameter $A(x, y, z, t)$ as

$$
\begin{aligned}
\langle A\rangle(t) & =\int \mathrm{d}^{3} x A(x, y, z, t) M(x, y, z, t) \\
& =\frac{1}{(2 \pi)^{3 / 2} \sqrt{8 t^{3} \kappa_{x x} \kappa_{y y} \kappa_{z z}}} \int \mathrm{d}^{3} x A(x, y, z, t) \mathrm{e}^{-\frac{x^{2}}{4 k_{x x}}-\frac{y^{2}}{4 k_{y y y}}-\frac{z^{2}}{4 k_{z z}}} \\
& =\frac{1}{(2 \pi)^{3}} \int \mathrm{d}^{3} k \mathrm{e}^{-\sum_{i=x, y, z} k_{i}^{2} \kappa_{i i} t} \int \mathrm{d}^{3} x A(x, y, z, t) \mathrm{e}^{-\mathrm{i} k x} .
\end{aligned}
$$

In turn we calculate the mean square deviation $\left(A=x_{i}^{2}\right)$ and the characteristic function $\left(A=\mathrm{e}^{\mathrm{i} \boldsymbol{k} \cdot \boldsymbol{x}}\right)$. 


\subsection{The mean square deviation}

Here we calculate the mean square deviation $\left\langle z^{2}\right\rangle$ in the parallel direction

$$
\begin{aligned}
\left\langle z^{2}\right\rangle & =\frac{1}{(2 \pi)^{3}} \int \mathrm{d}^{3} k \mathrm{e}^{-\sum_{i=x, y, z} k_{i}^{2} \kappa_{i i} t} \int \mathrm{d}^{3} x z^{2} \mathrm{e}^{\mathrm{i} k x} \\
& =\frac{1}{2 \pi} \int \mathrm{d} k_{z} \mathrm{e}^{-k_{z}^{2} \kappa_{z z} t} \int \mathrm{d} z z^{2} \mathrm{e}^{\mathrm{i} k_{z} z} \\
& =-\int \mathrm{d} k_{z} \mathrm{e}^{-k_{z}^{2} \kappa_{z z} t} \frac{\partial^{2}}{\partial k_{z}^{2}} \delta\left(k_{z}\right) \\
& =-\int \mathrm{d} k_{z} \frac{\partial^{2}}{\partial k_{z}^{2}} \mathrm{e}^{-k_{z}^{2} \kappa_{z z} t} \delta\left(k_{z}\right) \\
& =2 \kappa_{z z} t,
\end{aligned}
$$

which is the expected result, and we find that the width of the Gaussian function behaves diffusively. Because of the symmetric form of the Gaussian function, we obtain in general

$\left\langle x_{i}^{2}\right\rangle=2 \kappa_{i i} t$.

\subsection{The characteristic function}

The characteristic function is one of the key inputs into nonlinear transport theories (see e.g. Matthaeus et al. 2003; or Shalchi et al. 2004) and is defined as

$\Gamma(\boldsymbol{k}, t)=\left\langle\mathrm{e}^{\mathrm{i} \boldsymbol{k} \cdot \boldsymbol{x}}\right\rangle$

and therefore

$$
\begin{aligned}
\Gamma(\boldsymbol{k}, t) & =\frac{1}{(2 \pi)^{3}} \int \mathrm{d}^{3} k \mathrm{e}^{-\sum_{i=x, y, z} k_{i}^{2} \kappa_{i i} t} \int \mathrm{d}^{3} x \mathrm{e}^{\mathrm{i}\left(\boldsymbol{k}^{\prime}-\boldsymbol{k}\right) \boldsymbol{x}} \\
& =\int \mathrm{d}^{3} k \mathrm{e}^{-\sum_{i=x, y, z} k_{i}^{2} \kappa_{i i} t} \delta\left(\boldsymbol{k}^{\prime}-\boldsymbol{k}\right)
\end{aligned}
$$

and we finally obtain

$$
\Gamma(\boldsymbol{k}, t)=\mathrm{e}^{-\kappa_{x x} k_{x}^{2} t-\kappa_{y y} k_{y}^{2} t-\kappa_{z z} k_{z}^{2} t} .
$$

This result can be applied within nonlinear transport theories like the NLGC-approach of Matthaeus et al. (2003).

\section{Summary and conclusion}

In this paper an analytical investigation of the two-dimensional Fokker-Planck equation has been presented. The examination of this equation was split into three levels:

a) We explored the Fokker-Planck equation and the parallel diffusion coefficient for a general form of the pitch-angle Fokker-Planck coefficient to confirm the considerations of Earl (1974) in a more ostensive way.

b) By assuming a isotropic form of $D_{\mu \mu}$, a detailed investigation of the Fokker-Planck equation is presented. We calculated interesting parameters like the penetration depth. While the isotropic form of the Fokker-Planck coefficient disagrees with the quasilinear result, it can be confirmed by the recently derived second-order theory, if the turbulence is not too weak.

c) For sharp initial conditions, we derived simple formulas for all relevant parameters. This allows a visualization of the pitch-angle isotropization process, and it depicts the process of parallel transport of cosmic rays.

The results of this paper are important for understanding parallel transport and for the formulation of nonlinear theories. As described in the introduction, the characteristic function $\Gamma(\boldsymbol{k}, t)$ is the key input into nonlinear theories (see e.g. Matthaeus et al. 2003; Shalchi et al. 2004; Shalchi 2005a). Determination of this function can only be achieved if the particle distribution function is known. For example, if we consider the so-called slab model for the turbulence geometry (in this model the wave vectors are always parallel to the magnetic mean field) and assume a Gaussian form of the distribution function, we find for the characteristic function (see e.g. Shalchi 2005a)

$\Gamma\left(k_{\|}, t\right)=\mathrm{e}^{\mathrm{i} k_{\|}\langle z\rangle} \cdot \mathrm{e}^{\sigma_{z}^{2} / 2 \cdot k_{\|}^{2}}$

with the mean square deviation $\sigma_{z}^{2}$, which is dependent on both time-dependent parameters $\langle z\rangle(t)$ and $\left\langle z^{2}\right\rangle(t)$. In general, $\Gamma\left(k_{\|}, t\right)$ must be known for all time scales. Because the parameters $\langle z\rangle(t)$ and $\left\langle z^{2}\right\rangle(t)$ have been calculated in the current paper (see e.g. Eqs. (44) and (57)), the present work could be important for formulating nonlinear transport theories.

Furthermore, the time-dependent results presented here can be used to reproduce and understand test-particle simulations. Also for real astrophysical problems, time-dependent transport could be important; e.g. for very fast particles, the mean residence time could be very small. In this case the particle wouldn't have time to reach diffusive behavior and a timedependent description must be applied. There could also be cases where particle transport is non-diffusive for all timescales. A prominent example is perpendicular transport in the slab model (see e.g. Shalchi 2005b), where we find subdiffusive behavior. In such cases, a full time-dependent description of cosmic ray transport has to be accomplished.

Formulating accurate nonlinear transport theories, reproducing numerical test particle simulations, and knowing diffusion coefficients for all times scales are interesting and important in cosmic ray astrophysics.

\section{References}

Chandran, B. D. G. 2000, Phys. Rev. Lett., 85, 4656

Earl, J. A. 1974, ApJ, 193, 231

Jokipii, J. R. 1966, ApJ, 146, 480

Matthaeus, W. H., Qin, G., Bieber, J. W., et al. 2003, ApJ, 590, L53

Schlickeiser, R. 2002, Cosmic Ray Astrophysics (Berlin, Heidelberg: Springer-Verlag)

Shalchi, A. 2005a, Phys. Plasmas, 12, 052324

Shalchi, A. 2005b, JGR, 110, A09103

Shalchi, A., Bieber, J. W., Matthaeus, W. H., \& Qin, G. 2004, ApJ, 616,617 